\title{
The Effect of Abdominal Aortic Cross-Clamping on Myocardial Function
}

\author{
Ernest Dunn, M.D., Richard L. Prager, M.D., William Fry, M.D., \\ and Marvin M. KirSh, M.D. \\ Department of Surgery, Section of Thoracic Surgery and Section of General Surgery, The University \\ of Michigan Medical Center, Ann Arbor, Michigan 48109
}

Submitted for publication November 29, 1976

Elective aneurysmectomy and bypass grafting are now well established as the preferred methods of treatment for patients with symptomatic abdominal aortic aneurysms or abdominal aortic occlusive disease. Since these are late manifestations of generalized arteriosclerosis, it is not surprising that cardiac reserve is the major factor in determining operative survival. In an effort to define the myocardial response and peripheral vascular response to abdominal aortic operations more clearly, 27 patients undergoing elective abdominal aortic operations were studied.

\section{MATERIALS AND METHODS}

The 27 patients were divided into two groups: Group I (13 patients) underwent resection of abdominal aortic aneurysms. Group II (14 patients) underwent operation for aortoiliac occlusive disease.

Prior, during, and after operation, all patients had serial studies of cardiopulmonary dynamics based on a model developed by Siegel et al. [8]. Central venous as well as radial artery catheters were placed, utilizing the subclavian or internal jugular vein. Following these cannulations, notation was made of height, weight, arterial blood pressure, and central venous pressure. Prior to premedication for operation the patients underwent dye dilution cardiac output determinations to be used as a baseline. These basic data, height, weight, mean arterial pressure, central venous pressure, and calculation from the dye curve, were fed into a preprogrammed Olivetti 602 computer-calculator. The resulting information included the patient's cardiac output, cardiac index, stroke volume, total peripheral resistance, mixing time (a ventricular contractility index), dispersion time (a time index of blood flow through the small vessels of the lung), and mixing and dispersion volume (an estimate of volume in the heart and central circulation) [8].

The dye dilution studies were repeated at predetermined intervals during the observation period: (1) $3 \mathrm{~min}$ after anesthesia induction; (2) 1 to $3 \mathrm{~min}$ following crossclamping of the abdominal aorta; (3) 30 min following abdominal aortic crossclamping; (4) 1 to $3 \mathrm{~min}$ following removal of the aortic cross-clamp and first distal limb of the graft; (5) 1 to $3 \mathrm{~min}$ following removal of the second distal limb of the graft; (6) immediately upon entry into the recovery room; and (7) on the first postoperative day.

These eight observations were then compared, employing Student's $t$ test, between the Group I and Group II patients and among the members of the same group.

\section{EXPERIMENTAL RESULTS}

In Group I there were 3 women and 10 men, with an age range of 48 to 82 years. The average age was 65 . In Group II the sex ratio was more balanced, with 6 
men and 8 women. The age range in this group was 48 to 78 years with a slightly lower average age of 59. In an attempt to assess the degree of atherosclerotic cardiovascular disease, these groups were compared for evidence of preoperative congestive heart failure, angina, previous myocardial infarction, hypertension, and previous ccrcbral vascular accident. As Table 1 demonstrates, the incidence of previous myocardial infarction was higher in the patients with occlusive disease $(50 \%)$, as compared to $31 \%$ in the patients with aneurysms. The incidence of hypertension was also greater in the occlusive disease group, $43 \%$ compared to $31 \%$. Finally, two patients in the occlusive disease group had suffered a previous cerebral vascular accident, whereas none of the aneurysmal patients had had a previous stroke.

Preoperatively, hemodynamic measurements were similar in both groups. Following induction of anesthesia, which included an equal proportion of "balanced" " and halothane anesthesia in both groups, cardiac index fell at the same rate along with an increase in total peripheral resistance (Fig. 1 and 2). The mean pressure also dropped 5 to $7 \mathrm{~mm} \mathrm{Hg}$ in both groups (Fig. 3). A noticeable but statistically insignificant rise in mixing time occurred in both groups, indicating a compromise in ventricular contractility (Fig. 4). At clamp time (1), 1 to 3 min after cross-clamping the abdominal aorta, the drop in cardiac index continued and the total peripheral resistance rose noticeably in both groups. Mixing time rose in the patients with aneurysms and it leveled off in the patients with occlusive disease. The mean arterial pressure rebounded above baseline in both groups. While there was no significant difference between these two groups, the trend of impaired contractility, falling cardiac index and stroke volume, and rising total peripheral resistance showed significant differences between baseline values and clamp (1) in the subset of patients with aneurysms $(0.01<P<0.05)$, Table 2 . At clamp time (2), or the second observation period, during aortic cross-clamping, the cardiac index leveled off without showing a significant difference between the two groups. Total peripheral resistance remained higher in Group I although not significantly so between the groups. The rise above baseline within the group remained significant $(P<0.01)$. Mixing time remained significantly above baseline in the Group I patients $(P<0.01)$ and indeed was significantly higher in the Group I patients when compared to Group II patients, $(P<0.05)$ indicating continuing compromise in ventricular function.

With the release of the aortic and first distal clamp, the mean arterial pressure dropped slightly towards the preoperative level without significant hypotension.

TABLE 1

Manifestations of Atherosclerotic Disease in the Two Groups

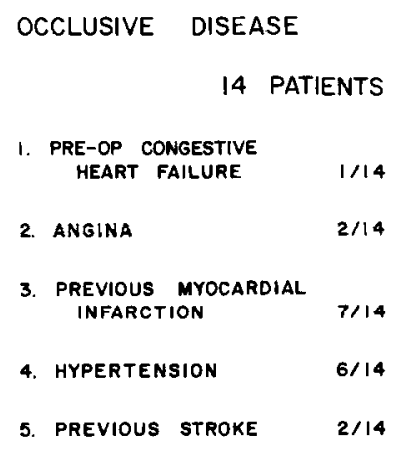

14 PATIENTS

I. PRE-OP CONGESTIVE HEART FAILURE I/14

2. ANGINA

$2 / 14$

3. PREVIOUS MYOCARDIAL
INFARCTION

4. HYPERTENSION

5. PREVIOUS STROKE

ANEURYSMAL DISEASE

13 PATIENTS

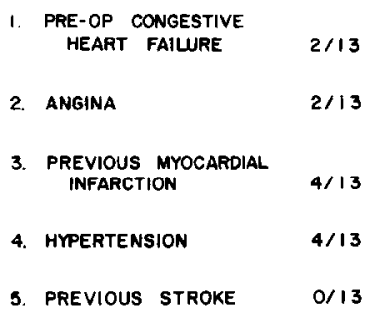




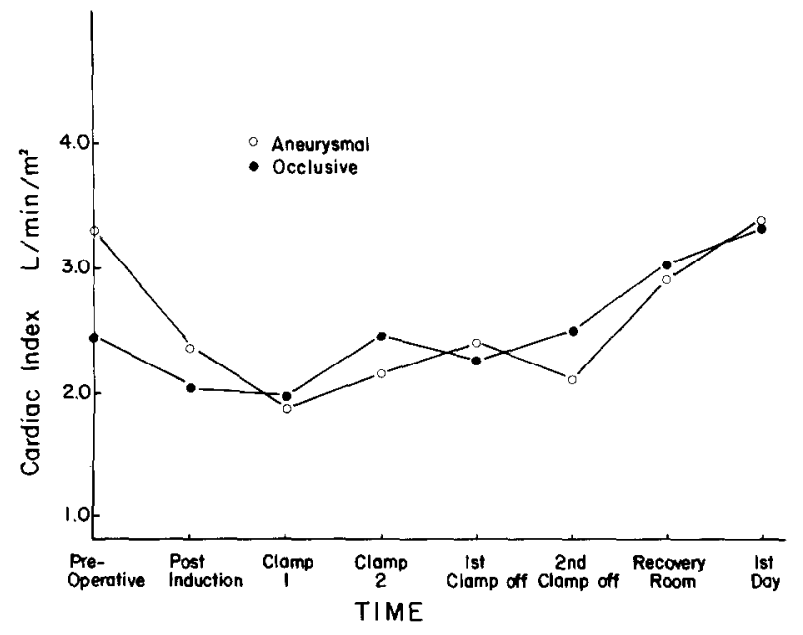

FIG. 1. Alterations in cardiac index in the two groups.

The cardiac index and stroke volume remained essentially unchanged as did the total peripheral resistance in the patients with occlusive disease, yet they dropped considerably in the patients with aneurysms. At $3 \mathrm{~min}$ after removal of the final clamp, "second clamp off," the mean arterial pressure in both groups dropped further. Cardiac index and total peripheral resistance were similar in both groups with a slight increase noted in Group I. Mixing time, although not significantly different, again increased in the aneurysm patients to equal the highest level.

In the recovery room there was a rebound recovery in mean arterial pressure with a further return toward baseline in cardiac index and stroke volume in both groups. Both total peripheral resistance and mixing time also fell toward the baseline. There was no significant difference between the groups in any measurement.

On the first postoperative day, the cardiac index, stroke volume, and mixing time re-

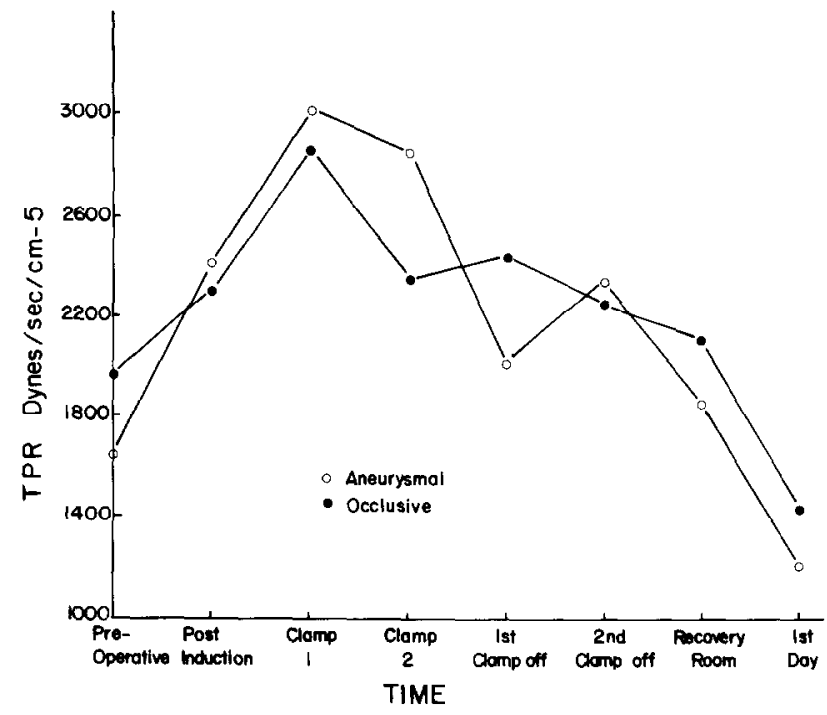

FIG. 2. Alterations in total peripheral resistance in the two groups. 


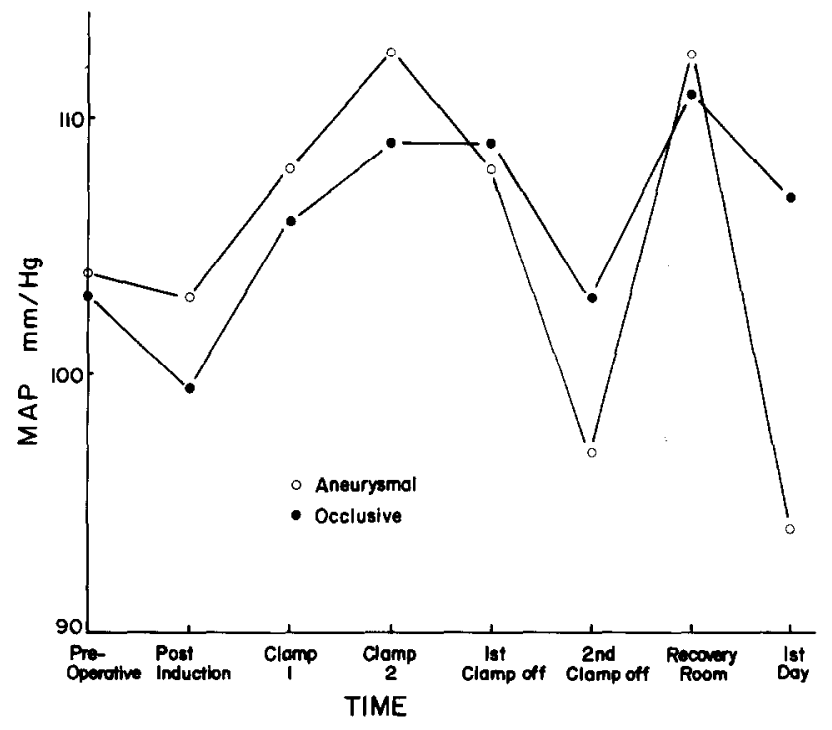

FIG. 3. Fluctuations in mean arterial pressure in the two groups.

turned to preoperative levels. Total peripheral resistance dropped below preoperative levels in both groups and the mean arterial pressure was lower in Group I.

There were no intraoperative or postoperative deaths in either group. Intraoperative blood loss and fluid replacement were comparable in each group as was clamp time. One patient in Group I was documented as having a subendocardial infarction by enzyme level measurements and electrocardiographic changes.

\section{DISCUSSION}

As a result of earlier diagnosis, refinements in operative techniques and prosthetic materials, and improvements in preand postoperative care, there has been a continued reduction in operative mortality

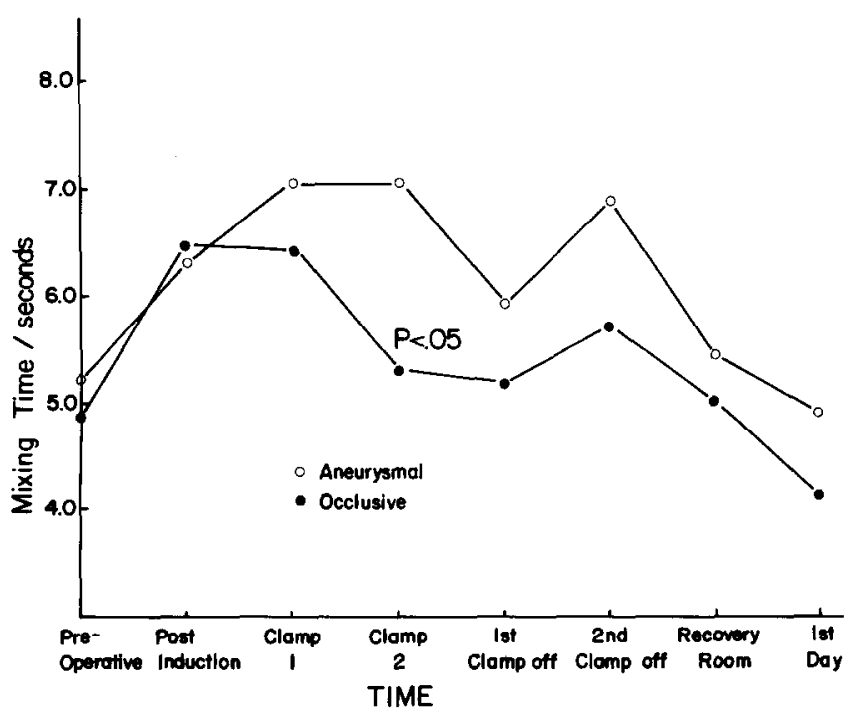

FIG. 4. Alterations in ventricular mixing time in the two groups. 
TABLE 2

Levels of Significance in the Group I Aneurysm Patients

\begin{tabular}{l|ccccccc}
\multicolumn{7}{c}{ ANFIRYSMAL DISEASE } \\
\hline TPR & Post & $\begin{array}{c}\text { Clamp } \\
\text { Clamp }\end{array}$ & $\begin{array}{c}\text { 1st } \\
\text { Clamp off }\end{array}$ & $\begin{array}{c}\text { 2nd } \\
\text { Clamp off }\end{array}$ & PARU & $\begin{array}{c}\text { 1st } \\
\text { Day }\end{array}$ \\
\hline CI & NS & $p<.01$ & $p<.01$ & NS & NS & NS & $p<.05$ \\
SV & NS & $p<.01$ & NS & NS & $p<.05$ & NS & NS \\
TIII & NS & $p<.05$ & $p<.01$ & NS & NS & NS & NS \\
MV & NS & NS & NS & NS & NS & NS & NS \\
MAP & NS & NS & NS & NS & NS & NS & NS
\end{tabular}

in patients undergoing abdominal aortic resections. In these patients the most significant factor affecting immediate survival are the sequelae of preexisting cardiovascular disease. Most reported scries find almost $50 \%$ of the postoperative deaths to be cardiac in origin, e.g., arrhythmia, myocardial infarction, and congestive heart failure $[2,6]$. The cardiovascular complication almost always occurred at the time of the infrarenal cross-clamping of the abdominal aorta.

Although much has been written concerning the etiology of and therapy for postclamp hypotension, very little has been said about hemodynamics during abdominal aortic occlusion. In 1968 Perry attempted to define more clearly the hemodynamics of abdominal aortic clamping by calculating cardiac output on 12 patients undergoing an abdominal aortic procedure [5]. He noted both increases and decreases in cardiac output. Because half of the deaths that do occur are related to myocardial dysfunction, this study was undertaken to define more clearly the myocardial and peripheral vascular changes that occur during abdominal aortic procedures.

Initial data from both groups show that the groups were similar in their baseline and initial hemodynamic measurements. Anesthesia precipitated a drop in cardiac index with a concomitant increase in total peripheral resistance in all patients, which continued during aortic clamping [7]. In Group I aortic clamping altered myocardial function and dynamics to a significant degree that was not observed in Group II. With a rise in resistance from 1700 to $3000 \mathrm{dyncs} / \mathrm{sec}^{-} / \mathrm{cm}^{-5}(P<0.01)$, a fall in cardiac index from 3.2 to $2.0 \mathrm{liters} / \mathrm{min} / \mathrm{m}^{2}$, and deterioration of ventricular contractility as the mixing time rose $(P<0.05)$, these patients reacted to cross-clamping with changes reflecting imminent myocardial decompensation. These changes persisted during clamping and gradually abated after clamping ceased. Unexpectedly, in the patients with occlusive disease who had a higher incidence of previous myocardial infarction, hypertension, and cerebrovascular injury, aortic cross-clamping produced a less impressive change in hemodynamic status. There were no significant changes from their preoperative levels compared to any observation period.

An additional difference was reflected in the mixing time which is an index of contractility. The patients with aneurysms showed a marked impairment of contractility compared to the patients with occlusive disease $(P<0.05)$. One can only speculate on the etiology of impaired contractility in these patients. Vincent and others have pointed out that the relationship between myocardial oxygen and available subendocardial blood supply is disrupted by left ventricular outlet obstruction 
[9]. Similarly, in the patients with aneurysms, the increase in total peripheral resistance from a probable absence of collateral vessels and the subsequent increase in afterload adversely affect the myocardial oxygen supply-demand ratio. Consequently, cardiac contractility worsened.

On the other hand, Group II, patients with a higher incidence of the ravages of atherosclerosis, did not demonstrate significant myocardial compromise. An increase in peripheral vascular resistance did not occur in these patients because they had an adequate network for collateral circulation.

Although no specific pharmacologic interventions were used in this study, there are several that might be considered for patients facing an acute increase in afterload who show myocardial decompensation. First of all, preoperative inotropic support in the form of digitalis has been suggested in those patients suspected to be at risk. Second, with the advent of vasodilator therapy for pump failure as described by Franciosa et al. [4] and recently supported by Chatterjee et al. [3], nitroprusside may offer an immediate method to decrease afterload. Recently, Attia et al. [1] and Carroll et al. [2] reported patients whose hemodynamic status was improved during aortic cross-clamping utilizing nitroprusside.

Our study indicates that patients without prior occlusive disease, who are undergoing resections for abdominal aortic aneurysms, are subject to altered myocardial function during aortic cross-clamping. While further research on management of this condition is needed, it may be that pharmacologic intervention, both preoperative and intraoperative, will lessen the myocardial compromise and improve overall survival.

\section{SUMMARY}

This study of 27 patients undergoing abdominal aortic operations was under- taken in an effort to define myocardial and peripheral vascular responses more precisely. Thirteen patients underwent resection of abdominal aortic aneurysms and fourteen patients underwent operation for aortoiliac occlusive disease. Both groups of patients demonstrated falls in cardiac index with a rise in total peripheral resistance following anesthesia and aortic crossclamping. The patients undergoing aneurysmal resection demonstrated a persistent altered myocardial function that was not observed in the occlusive disease patients. This difference may reflect the inability of the patients with aneurysmal disease or an extensive collateral network to handle the acute increase in afterload placed upon the myocardium.

\section{REFERENCES}

1. Attia, R., et al. Myocardial ischemia due to infrarenal aortic cross-clamping during aortic surgery in patients with severe coronary artery disease. Circulation 53: 6, 1976.

2. Carroll, R., Laravuso, R., and Schauble, J. F. Left ventricular function during aortic surgery. Arch. Surg. 3, 740, 1976.

3. Chatterjee, K., et al. Effects of vasodilator therapy for severe pump failure in acute myocardial infarction on short-term and late prognosis. Circulation 53: 5, 1976.

4. Franciosa, J., et al. Improved left ventricular function during nitroprusside infusion in acute myocardial infarction. Lancet 1: 650, 1972.

5. Perry, M. The hemodynamics of temporary abdominal aortic occlusion. Ann. Surg. 168: 2, 1968 .

6. Subawala, P., Strong, M. J., and Keats, A. S. Surgery of the aorta and its branches. Anesthesiology 33: 2. 1970.

7. Siegel, J. H. The myocardial contractile state and its role in the response to anesthesia and surgery. Anesthesiology 30: 5, 1969.

8. Siegel, J. H., et al. Quantifying the need for cardiac support in human shock by a functional model of cardiopulmonary vascular dynamics: With special reference to myocardial infarction. J. Surg. Res. 13: 4, 1972.

9. Vincent, W., Buckberg, G., and Hoffman, J. Left ventricular subendocardial ischemia in severe valvar and supravalvar aortic stenosis: A common mechanism. Circulation 49: 326, 1974. 